\title{
Diffraction limited imaging in the visible from large ground-based telescopes: new methods for future instruments and telescopes
}

Craig Mackay, Nick Law, Timothy D. Stayley

Craig Mackay, Nick Law, Timothy D. Stayley, "Diffraction limited imaging in the visible from large ground-based telescopes: new methods for future instruments and telescopes," Proc. SPIE 7014, Ground-based and Airborne Instrumentation for Astronomy II, 70141C (9 July 2008); doi:

10.1117/12.787439

Event: SPIE Astronomical Telescopes + Instrumentation, 2008, Marseille, France 


\title{
Diffraction Limited Imaging in the Visible from Large Ground-Based Telescopes: New Methods for Future Instruments and Telescopes
}

\author{
Craig Mackay $^{*}$, Nick Law ${ }^{\mathrm{b}}$, Timothy D. Stayley ${ }^{\mathrm{a}}$ \\ anstitute of Astronomy, University of Cambridge, Madingley Road, Cambridge UK, CB3 0HA \\ ${ }^{b}$ Department of Astronomy, Mail Code 105-24, California Institute of Technology, 1200 East \\ California Blvd., Pasadena, CA 91125, USA
}

\begin{abstract}
Faint object diffraction limited imaging in the visible from the ground has recently been demonstrated on a $5 \mathrm{~m}$ telescope with more than twice the resolution of Hubble for the first time. It has shown the way towards diffraction limited imaging in the visible with the next generation of large telescopes. This paper describes the results of experiments to show how this is achieved and what is needed to work well with faint natural guide stars. The importance of a large isoplanatic patch size is also emphasised. In particular, we will describe a new approach to the design of high efficiency, low order adaptive curvature sensors which use photon counting CCD detectors. Such systems used on larger telescopes together with image segmentation and resynthesis techniques using closure phase techniques are shown to have an important place in achieving these goals. The optimum combination of these different techniques will be explained for a variety of different applications.
\end{abstract}

Keywords: Lucky Imaging, Electron multiplying CCDs, EMCCDs, Curvature sensors, Adaptive optics

\section{Introduction}

Lucky Imaging ${ }^{1}$ is now established as an effective and efficient technique of achieving Hubble resolution $(\sim 0.1 \operatorname{arcsec})$ in I- band on $2.5 \mathrm{~m}$ class telescopes on good astronomical sites. The technique consistently shows much larger isoplanatic patch sizes than are found with adaptive optics techniques by a factor of about 10 . The development of virtually noise free back-illuminated electron multiplying CCDs by E2V Technologies (Chelmsford, UK) with photon counting performance ${ }^{2}$ has been critical to the technique. Our goals are simply to achieve significantly higher resolution imaging in the visible from the ground band than is delivered by Hubble. We believe it is critical to develop a technique that can use faint natural guide stars that have a high enough sky density to give a high probability of being present in most parts of the sky. There are big advantages in being able to use natural guide stars for these techniques because they will give the best correction. The isoplanatic patch size we find with Lucky Imaging makes this a promising route to explore.

Higher resolution imaging requires either a larger telescope or a shorter wavelength. Even on the best astronomical sites the seeing is seldom good enough to make this an efficient observing strategy with telescopes larger than $\sim 2.5 \mathrm{~m}$ diameter. The probability of getting a near diffraction limited image becomes vanishingly small rather quickly as telescope diameter increases. Adaptive optics techniques either on their own or in conjunction laser guide stars are now working reasonably well in the near infrared but have been much less successful in the visible. The best systems have now barely exceeded the resolution of the Hubble Space Telescope.

A different approach is necessary if we are to improve our luck enough to make high resolution imaging practical with good efficiency on telescopes in the 5-10 m class. One approach has recently been tried on the $5 \mathrm{~m}$ telescope on Mt.

* e-mail:cdm@ast.cam.ac.uk; phone +44-(0)1223 337543; fax +44-(0)1223 330804

Ground-based and Airborne Instrumentation for Astronomy II, edited by lan S. McLean, Mark M. Casali, Proc. of SPIE Vol. 7014, 70141C, (2008) · 0277-786X/08/\$18 · doi: 10.1117/12.787439 
Palomar in California. Here we used the PALMAO adaptive optics system in front of our Lucky camera. With a bright reference star in the field, PALMAO was able to substantially reduce the power on the largest turbulent scales so that the Lucky Imaging camera could concentrate its efforts on smaller turbulence scales. The results obtained were surprisingly good ${ }^{3}$. With fast image selection behind the Palomar AO system we obtained Strehl ratios of 5-20\% at 700 $\mathrm{nm}$ in a typical range of seeing conditions, with a median Strehl of approximately $12 \%$ when $10 \%$ of the input frames are selected. At wavelengths around $700 \mathrm{~nm}$ the system gave diffraction-limited 35 milliarcsecond FWHMs. At $950 \mathrm{~nm}$ the output Strehl ratio was as high as $36 \%$ and at $500 \mathrm{~nm}$ the FWHMresolution was as small as 42 milliarcseconds, with a low Strehl ratio but resolution improved by factor of $\sim 20$ compared to the prevailing seeing. To obtain wider fields we also used multiple Lucky-Imaging guide stars in a configuration similar to a ground layer adaptive optics system. With eight guide stars but very undersampled data we obtained 300 milliarcsecond resolution across a $30 \times 30$ arcsec field of view in i' band.

Some of the images that were taken during this run are the highest resolution images ever taken in the optical or near infrared by any single telescope, from ground or from space. An example from the globular cluster M13 is shown in figure 1.
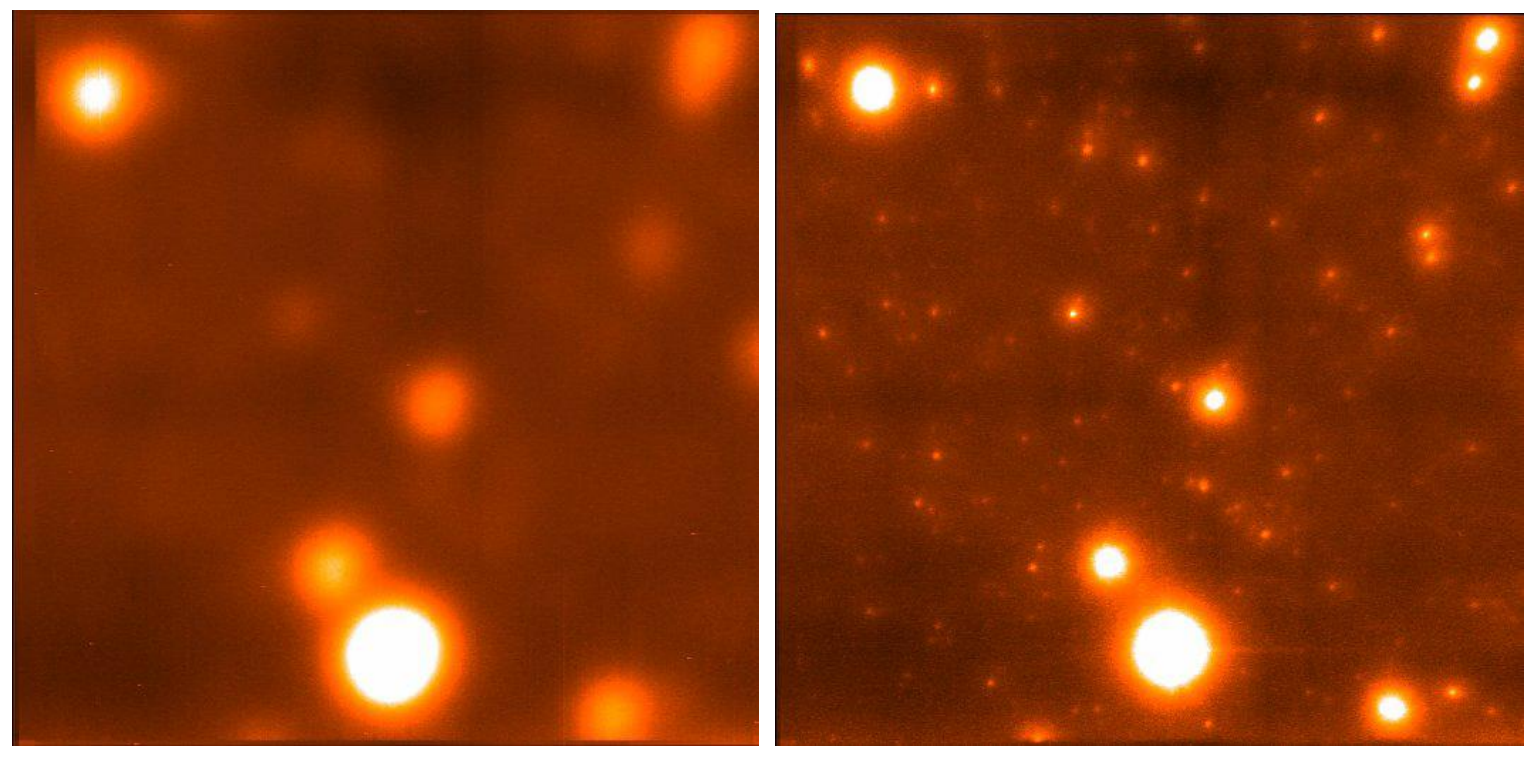

Figure 1: The Globular cluster M13 as imaged conventionally by the Palomar $5 \mathrm{~m}$ telescope (left), and M13 as imaged with the Lucky Camera behind the PALMAO adaptive optics system on the Palomar $5 \mathrm{~m}$ telescope (right). The resolution obtained on this I-band pair of images was improved from the 0.65 second natural seeing to about 35 milliarcseconds, approximately 3 times better than that ever achieved from the Hubble Space Telescope ${ }^{3}$.

\section{High Resolution Lucky Imaging with Faint Reference Stars}

The relatively low order adaptive optic system that was used on Palomar described above used a high-speed ShackHartmann sensor as wavefront sensor. Such an approach requires a relatively bright reference star, with a magnitude that limits the applicability of that method to a very small fraction of the sky. For Lucky Imaging purposes we do not need high order correction. Rather we would prefer a lower order wavefront sensor that measured the wavefront errors with faint guide stars. With the Shack-Hartmann sensor, as soon as the reference star brightness gets below some limit (typically 25 detected photons per frame) in each cell it simply stops working. In contrast a curvature sensor can derive useful wavefront error information even at rather faint levels. The curvature sensor anyway seems, in practice, to work at fainter levels particularly for low order sensors. A study by Racine ${ }^{4}$ has shown that curvature sensors deployed on telescopes today are able to work at a level about 2.5 magnitudes fainter than Shack-Hartmann sensors under similar conditions. 
In the past curvature sensors have proved significantly harder to construct. The principle of the curvature sensor is that while the illumination in the pupil plane is completely uniform, on either side the wavefront it shows intensity variations that are directly related to the phase errors in the wavefront. By comparing the intensities on either side of the pupil plane it is possible to derive directly a measure of the wavefront curvature. That may then be used to drive a flexible correction mirror that feeds the science instrument. The number of cells that one needs for a curvature sensor is very much smaller than for a Shack-Hartmann sensor. The shapes of these cells, however, are ideally rather complex so that lenses cut to shape have to be made. The more successful sensors have used avalanche photodiodes, which have their own problems in terms of quantum efficiency and reliability. These have been fed directly from the shaped lenses.

The development in recent years of high quantum efficiency photon counting high-speed electron multiplying CCDs has opened the possibility of recording these near-pupil plane images as two-dimensional images which are then processed in real-time to derive the curvature information needed to drive a flexible mirror. The configuration of the cell sizes and shapes is then done entirely on the computer and therefore can be easily changed in response to changing observing conditions or observing wavelengths. There is a useful and comprehensive review of the advantages and application of curvature sensors by Guyon et al. ${ }^{5}$ which shows that it is possible to get very close to the theoretical maximum performance by using the correct approach to designing a curvature sensor.

Guyon et al. ${ }^{5}$ also show that with careful design it is possible to use relatively broad band-passes because things may be arranged to suffer from a very little chromaticity. Our simulations suggest that with careful design it should be possible to produce a low order correction as we need for Lucky Imaging on an $8 \mathrm{~m}$ class telescope that will work effectively on a guide star with $\mathrm{I}=18-18.5$. This increase sensitivity comes from partly from using a deep-depletion CCD as the curvature sensor and the fact that for low-order sensing it is possible to run the wavefront sensor more slowly. On larger telescopes the temporal phase decorrelation is slower. The very high frame rates used with high-order ShackHartmann sensors are not necessary here. In addition the measured wavefront errors on any frame gave an excellent image quality indication that we may use to inform our Lucky Imaging selection procedure. At these guide star magnitudes the chance of finding a suitable reference star becomes relatively high, particularly when coupled with the relatively large isoplanatic patch size that we find routinely with Lucky Imaging.

\section{High Resolution Lucky Imaging on Next-Generation Large Telescopes.}

As we found previously there will be limits to what can be achieved with a simple low order adaptive optics system followed by a Lucky Imaging camera no matter how carefully it is designed. If we are to work at shorter wavelengths or on even larger telescopes then again the chance of getting a sharp enough image becomes vanishingly small. In principle one could use a higher order adaptive optics system but quickly the brightness necessary for an acceptable signal-to-noise reference star becomes much higher since higher-order implies faster read out and a higher resolution to the derived wavefront errors. As before we need to find some other way of increasing our luck.

One way of achieving this is to reduce the area of the telescope over which we collect the light. With a segmented mirror telescope such as Keck we can imagine directing groups of segments (for example four at a time: see Figure 2) towards one detector. The image of the sky on that detector is then that formed by a single segment with each compact object crossed by Michaelson interference fringes that represent the spacing between the segments being combined. As before we assume that there is still a low order adaptive optics system in place to remove the larger scale turbulent errors. If we choose the total area of the segments to be comparable to the area of a single telescope that gives an acceptable degree of "luck" then we will see the fringe visibilities coming and going as the turbulence changes. Now the Lucky Imaging involves selecting those moments with high fringe visibilities.

Closure phase techniques developed by radio astronomers and, more recently used by optical astronomers in groundbased interferometers allows the fringe patterns to be processed to give high resolution images. For an unresolved object, the relative phases of each of the fringe patterns around in a triangle should add to zero. Even when the fringe visibilities are reduced the closure phase is unaffected in terms of its value. It is only affected in terms of signal-tonoise. The relative phases of these fringe components give the relative phases of the Fourier components of the twodimensional sky brightness distribution in the region of the object. In radio astronomy interferometry, large-scale structures are resolved out but here large-scale structures are unaffected. They do not produce any fringe information but their photons are still detected with full efficiency so large-scale, diffuse structures are not attenuated in any way. 

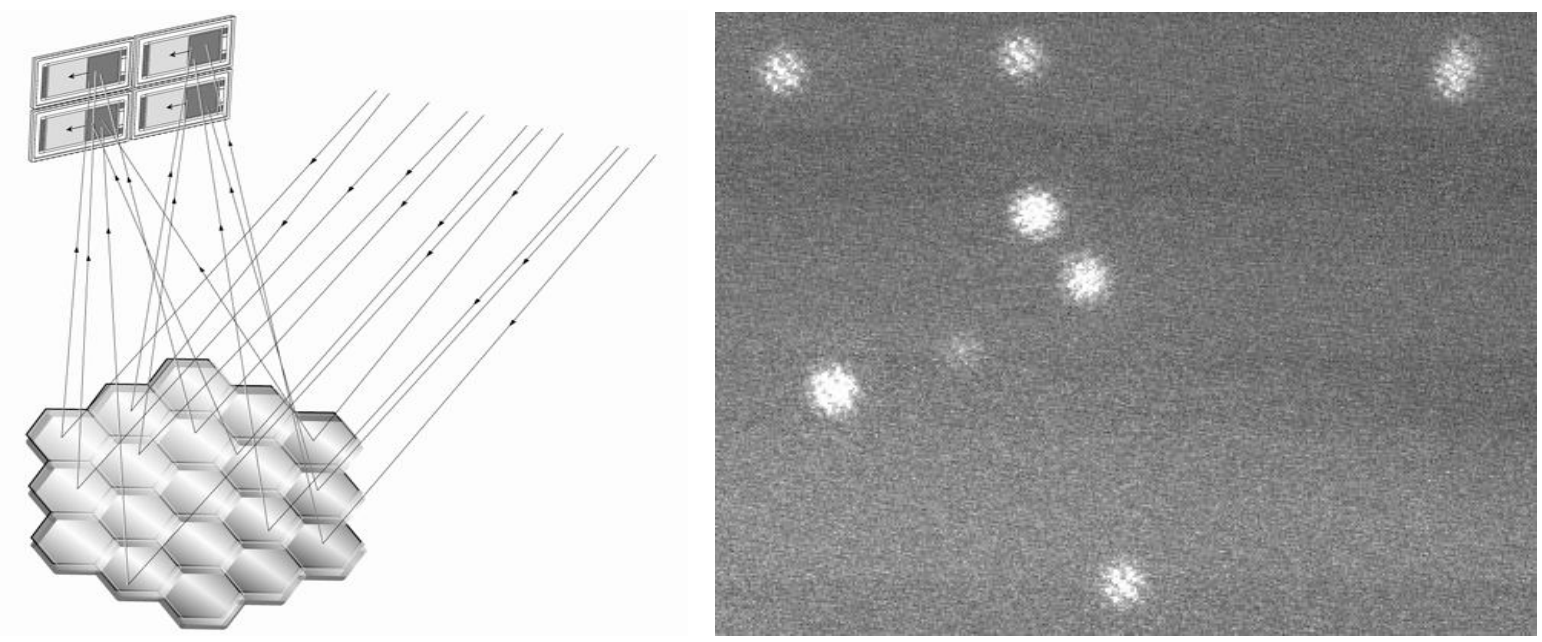

Figure 2: Conceptual outline of the instrument. Each star is detected as it would be if imaged by one sub-aperture, but now crossed by Michaelson interference fringes from the spacings of the sub-apertures. The fringe peaks coincide, showing the closure phase here is zero. Each star has identical fringe patterns across it. This image was taken in the laboratory, showing instrumental diffraction limited performance on an artificial globular cluster, at $400 \mathrm{~nm}$

By combining other groups of four segments on to other detectors each of which has its own moments of high visibility fringes, this system will be able to derive the relative phases of all the Fourier components needed to synthesise a full resolution image. By using all the segments the full light-gathering power of the telescope aperture is used. In many cases it will be more convenient to use an intermediate segmented mirror to break up the aperture of the telescope behind the low order adaptive optics system. Tests have been carried out with the $3.6 \mathrm{~m}$ NTT telescope at La Silla, Chile to demonstrate these techniques. Although the system used did not have any atmospheric dispersion corrector or any adaptive optics capability it was still possible to demonstrate that high resolution fringes could be obtained even at short wavelengths. At a wavelength of $450 \mathrm{~nm}$ the resolution of a $3.6 \mathrm{~m}$ telescope is about 30 milliarcseconds and relatively high visibility fringes were obtained as expected.
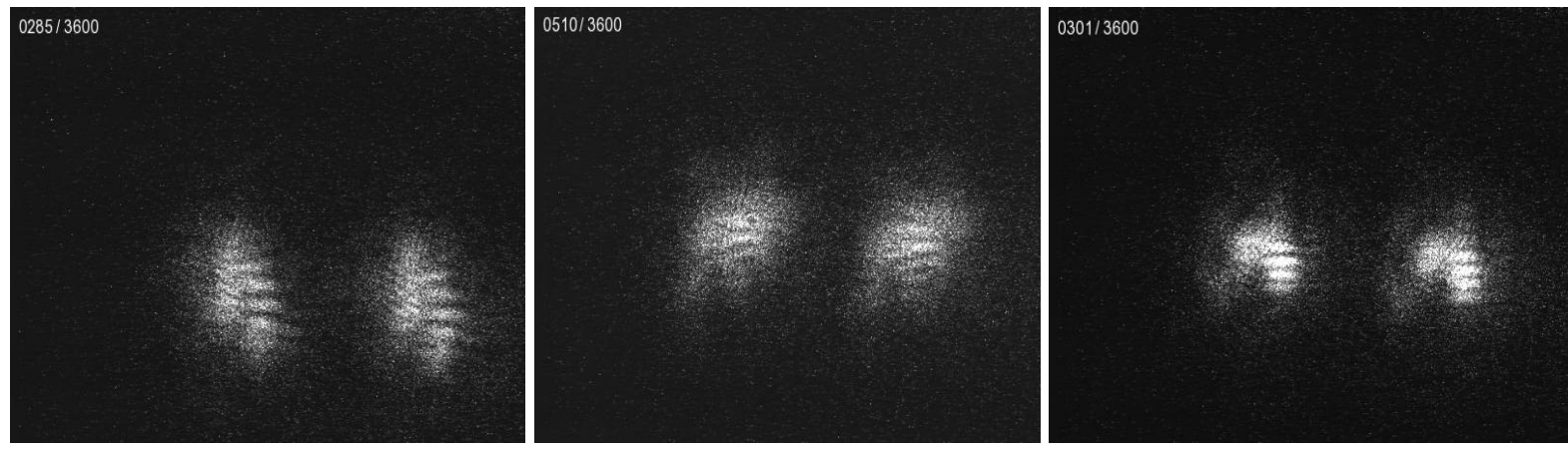

Figure 3: Three relatively well aligned frames from a sequence of a double star, about two arc second separation and each about $12 \mathrm{mag}$ in B $(450 \mathrm{~nm})$. Four apertures of $60 \mathrm{~cm}$ diameter give six fringe baselines. The correlation between the fringe patterns on the two stars is excellent. The alignment of the four beams is relatively poor, demonstrating the importance of having low order correction on each of the beams to assure consistent alignment and therefore the highest possible fringe visibility. 


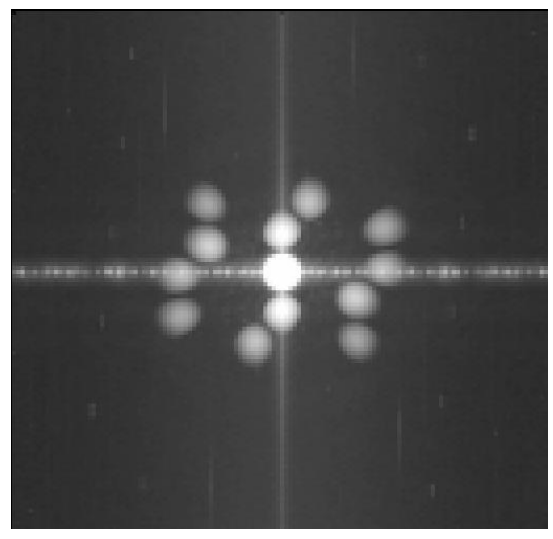

Figure 4: This image shows the summed power spectrum of a sequence, frames of which are shown in Figure 3. Power from each of the six baselines are clearly visible (the power spectrum shows each baseline twice) up to a resolution of 30 mas.

Predictions are that we should be able to achieve near diffraction limited imaging in I band on an $8 \mathrm{~m}$ telescope using the full aperture with a low order adaptive optics system. For larger telescopes or shorter wavelengths operation it will be necessary to use an aperture segmentation approach in order to improve the level of luck to give an acceptable observing efficiency.

\section{Instrumental Considerations for Adaptive Optics Plus Full Aperture Imaging}

The successful implementation of the instruments described here requires a number of components to be integrated in a novel manner. The curvature sensor will follow the concepts described by Guyon et al. ${ }^{5}$ and use four different conjugation planes, two on either side of the pupil plane to allow the use of non-linear Fourier-based wavefront reconstruction algorithms. The sensor will use out-of-band light divided four ways to give four pupil plane images onto a single 512 x 512 electron multiply back illuminated CCD. It will be operated at a pixel rate in the range of 15-25 $\mathrm{MHz}$ to give a frame rate of 60-100 frames per second in photon counting mode. The pupil will be imaged on to a field about 200 pixels in diameter giving very high resolution detection and photon rates per pixel low enough to enable thresholding and photon counting (and therefore the full quantum efficiency of the electron multiply CCD) even on relatively bright stars.

The data from the curvature sensor CCD will be processed in real time and the data so generated will be used to control a compact deformable mirror such as that manufactured by Boston Micromachines Corporation ${ }^{6}$. This deformable mirror uses MEMS technology and has an array of $12 \times 12$ actuators. In addition to controlling the deformable mirror the data from the curvature sensor will give an image quality assessment. This will be used to guide the Lucky Imaging selection. One of the weaknesses of the Lucky Imaging strategy generally is that if you want to use a narrow pass-band then Lucky selecting on the science images becomes dependent on the availability of a rather bright reference star in the field of view. By using a narrow band filter for the science camera but using a much broader band for the curvature sensor using the out-of-band light allows efficient narrow band Lucky Imaging to be achieved with fainter reference stars.

Electron multiply CCD detectors with high quantum efficiency (back-illuminated) are only available at present in sizes up to 1024 x 1024 pixels. When working at high resolution this means that the field of view is very limited. With an $8.2 \mathrm{~m}$ telescope in I band the diffraction limit is about 24 milliarcseconds. To sample this limit correctly requires pixels 8-10 milliarcseconds square giving a field of view that is only 8-10 arc seconds across. We find that the isoplanatic patch size with Lucky Imaging is significantly larger than this and restricting the field to be as small as this can significantly reduce the probability of finding a satisfactory reference star within the field of view. In order to increase the field of view we propose to use an array of four CCDs each 1024 x 1024 pixels. Electron multiply CCDs are not at present buttable since there are significant areas on the CCD around a sensitive area that are needed for clocking tracks. 
We propose to use a shallow angle pyramid very similar in structure to that used on the Wide Field/Planetary camera on the Hubble Space Telescope. The pyramid is positioned close to the focal plane with the field divided into four at the pyramid. The now diverging beams are reflected and re-imaged on to the individual separate CCDs in a liquid nitrogen cooled vacuum dewar. The four CCDs are run at up to $25 \mathrm{MHz}$ pixel rate giving a frame rate of $25 \mathrm{~Hz}$. Higher frame rates may be achieved by limiting the amount of area read out of the CCD.

The data are captured by high-speed digital interface cards working on the PCI-Express bus. Real-time Lucky Image processing is essential with this configuration because of the very large amount of data that the instrument produces (over 200 MB per second). These data volumes are greatly in excess of what may be handled by a single PC processor. However the Lucky Imaging process is something which is highly amenable to accelerating with a multiprocessor parallel approach. We are beginning to develop software that uses the NVIDIA Tesla ${ }^{7}$ special processor card that can, under ideal conditions, give as much as 400 times the throughput of a single PC. Such a level of processing power is considerably more than we think we need but it is very difficult to be sure of this until coding is much more advanced.

\section{Instrumental Considerations for Adaptive Optics Plus Segmented Mirror Lucky Imaging}

The great majority of the components of the system will be the same as those used and described in section 4 . The simplified conceptual layout of this configuration is shown in Figure 5.

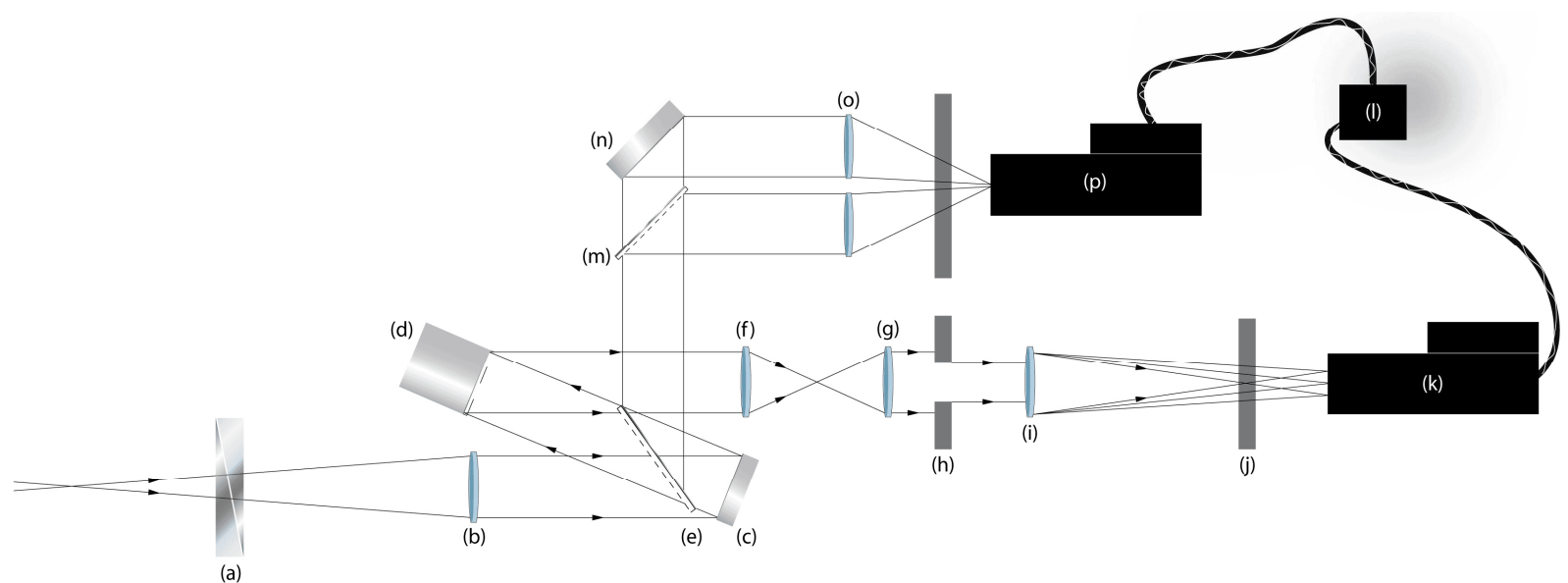

Figure 5: The beam from the telescope enters from the left of the figure and passes through focus. The first active component is an atmospheric dispersion corrector (a) which is essential for high-resolution imaging away from the zenith if an acceptably wide bandwidth is to be used. A collimating lens (b) generates a pupil plane close to the fold mirror (c). Mirror (c) is the deformable MEMS unit controlled by the curvature wavefront sensor. The MEMS sensor is mounted on a simple tip-tilt mechanism to minimise the stroke required from the MEMS unit. A dichroic at (e) takes out of band light and sends it to the curvature wavefront sensor. The diagram shows light would be divided into two beams rather than four: see text). Mirror (m) reflects half the light and mirror (n) reflects the remainder. Lenses at (o) are adjusted to focus either side of the pupil plane to produce side-by-side images through a filter wheel on to an electron multiplying CCD detector (p). An in-band light pupil is created at mirror (d) using simple lenses that are omitted on this diagram for clarity. The mirror (d) is segmented into a series of sub apertures. The sub aperture defining mirrors are mounted on Piezo deflectors capable of three axis motion. This allows us to manage any telescope flexure as it moves around the sky which would normally be acceptable for conventional imaging work but are too great if we are to achieve true diffraction limited imaging. Not shown on the diagram is an aperture mask designed to control and vary the equivalent size of the sub apertures on the telescope depending on the wavelength in use and the conditions on the night in question. The piezo deflectors also allow us to phase the 16 beams correctly so that the necessary Michaelson interference fringes are obtained simultaneously. The aligned beams are then focussed (to baffle the field of view) and recollimated before passing through a long focus lens (i) which then forms four separate images on the four $\mathrm{CCD}$ detectors at position $(\mathrm{k})$ after the light has passed through a filter wheel at position $(\mathrm{j})$. The four CCD detectors are mounted inside a liquid nitrogen cooled dewar $(\mathrm{h})$. The output from the camera electronics is taken to the host computer (l) and a frame grabber/digital signal processor card to handle the processing in real time. 
The principal difference in this higher-resolution configuration is the need to segment the aperture. This is done by creating a pupil plane within the instrument on the mirror that segments and deflects the images from different parts of the aperture onto the different detectors. With only four detectors it can be difficult to fully fill the aperture but as this is a technology demonstrator more than anything else we expect this to be the best way to achieve much higher resolution. Again one of the issues is the field of view of the instrument. On an $8.2 \mathrm{~m}$ telescope in B Band the diffraction limit of the telescope will be about 13 milliarcseconds requiring 4-5 milliarcsecond scale per pixel. This will give a field of view of only 4-5 arc seconds. It is clear that the use of these imaging methods will create a considerable demand for much larger area electron multiplying CCDs with high quantum efficiency.

The image processing and Lucky Imaging selection necessary for this is going to be significantly more demanding than for the instrument described in section 4. Again we expect to need parallel processing assistance in order for real-time operation to be routinely practical.

\section{Conclusion}

We have already demonstrated the highest resolution images ever taken on single dish optical or infrared telescopes by using low order adaptive optics in front of a lucky imaging camera. The resolution obtained was approximately 3 times that achieved with the Hubble Space Telescope. There is considerable demand from astronomers for much higher resolution imaging facilities on ground-based telescopes, particularly in the visible. Current adaptive optics instruments even when used in conjunction with laser guide stars prove to be difficult to use and struggle to equal Hubble resolution far less exceed it. The instruments described above show offer every prospect of being able to deliver an exciting step in the angular resolution that may be obtained on ground-based telescopes. Some of the technologies required will be interesting to develop but do, at present, appear to be entirely practical.

\section{Acknowledgements}

We should like to acknowledge useful discussions with John Baldwin and Peter Warner of the Cavendish Laboratory, Cambridge University, and with Olivier Guyon, Subaru Telescope. We particularly thank the PALMAO team, especially Jenny Roberts and Antonin Bouchez, for all their help during the design, setup and operation of the instrument. Thanks also go to the Palomar Observatory team for great assistance throughout our run.

\section{References}

1. Baldwin, J.E, Warner, P.J., and Mackay, C.D., "The Point Spread Function in Lucky Imaging and Variations in Seeing on Short Timescales", (2008), Astron \& Astrophys, vol. 480, p589,2008

2. Lucky Imaging Website is at http://www.ast.cam.ac.uk/ optics/Lucky_Web_Site/index.htm which contains a lot of information about lucky imaging and the photon counting detector is that are used for it.

3. R. Racine, "The Strehl Efficiency of Adaptive Optics Systems", (2006), Pub. Astr. Soc Pacific, vol 118, p10661075.

4. N.M. Law, C.D. Mackay, et al, "Getting Lucky with Adaptive Optics: Fast AO Image Selection in the Visible with a Large Telescope" (2008) (submitted to astrophysical journal, May 2008)

5. Olivier Guyon et al, "Improving the sensitivity of Astronomical Curvature Wavefront Sensor using dual-stroke curvature" (2008), PASP, in press.

6. Boston Micromachines Corporation: see: http://www.bostonmicromachines.com

7. NVIDIA Tesla card: see: http://www.nvidia.com/object/tesla_c870.html 